\title{
EVALUACIÓN SÍSMICA DE CONSTRUCCIONES HISTÓRICAS DE MAMPOSTERÍA: COMPARACIÓN DE TRES MODELOS DE ANÁLISIS
}

\author{
Agustín Orduña Bustamante ${ }^{(1)}$, Guillermo Roeder Carbo $^{(1)}$ y Fernando Peña Mondragón ${ }^{(2)}$
}

\begin{abstract}
RESUMEN
En este artículo se describen brevemente tres procedimientos de análisis que se han propuesto para representar numéricamente la respuesta sísmica de construcciones históricas de mampostería: Análisis Límite con Bloques Rígidos, el Método de los Elementos Rígidos, y el Método del Elemento Finito no lineal. Estos tres métodos se aplican al análisis de una subestructura de un edificio histórico sujeto a cargas laterales, y que fue ensayado experimentalmente por otros investigadores. Se comparan las respuestas de los modelos numéricos con la del modelo experimental. Se observan similitudes aceptables de las respuestas de los cuatro modelos.
\end{abstract}

\begin{abstract}
This paper briefly describes three analytical procedures, which have been proposed for the numerical representation of the seismic response of ancient masonry constructions: Limit Analysis with Rigid Block models, the Rigid Element Method, and the non-linear Finite Element Method. These three methods are applied to the analysis of a substructure of a historical building, experimentally tested by other authors. The behaviours predicted by the numerical models are compared against the experimental model, and similar responses are observed.
\end{abstract}

\section{INTRODUCCIÓN}

En distintas partes del mundo se conserva un gran número de monumentos antiguos de mampostería que han sobrevivido durante los tiempos a diversas solicitaciones. De particular interés en este artículo están aquellas construcciones sujetas repetidamente a acciones sísmicas. El hecho de haber resistido estos eventos no implica que estén libres de peligro, como lo demuestran los daños producidos en edificios históricos por los sismos de Umbria-Marche, Italia en 1997 y 1998, en Puebla y Turquía en 1999, o en Bam, Irán en 2003.

Históricamente, el empleo de la mampostería como material de construcción ha sido muy importante, particularmente hasta antes del uso del concreto reforzado y el acero. De tal manera que un porcentaje muy importante de las construcciones con valor patrimonial están hechas de este material, tanto en México como en el resto del mundo. Por ello es de especial importancia el estudio estructural de construcciones de mampostería sujetas a acciones sísmicas.

Artículo recibido el 3 de abril de 2006 y aprobado para su publicación el 2 de noviembre de 2007. Se aceptarán comentarios y/o discusiones hasta cinco meses después de su publicación.

(1) Facultad de Ingeniería Civil, Universidad de Colima, km 9, carr. Colima-Coquimatlán, CP 28400, Coquimatlán, Col. aord@ucol.mx; groederc@ucol.mx

${ }^{(2)}$ Instituto de Ingeniería, UNAM, Ciudad Universitaria, CP 04510, México, DF, fpem@pumas.iingen.unam.mx 
El diseño y construcción de los edificios que hoy son parte del patrimonio cultural de la humanidad, fue posible gracias a la acumulación de conocimientos empíricos, que generalmente fueron transmitidos en secreto de generación en generación. En contraste, actualmente se cuenta con conocimientos, técnicas avanzadas de análisis y poderosas herramientas de cálculo automático que permiten modelar numéricamente, con gran precisión, el comportamiento mecánico de estructuras complejas. Sin embargo, el análisis estructural de edificios históricos de mampostería con fines de evaluación sísmica continúa siendo un desafío debido a una serie de factores. Entre ellos se cuentan: (a) la baja resistencia en tensión con falla casi-frágil de la mampostería, que implica comportamiento no lineal desde intensidades de carga pequeñas; (b) la gran heterogeneidad generalmente presente en estas construcciones dificulta asignar propiedades mecánicas globales al modelo del material; (c) el carácter compuesto del material; (d) la falla por corte es no asociada, debido a la diferencia entre el ángulo de dilatancia y el ángulo de fricción interna de la mampostería; (e) las juntas comúnmente representan planos de debilidad que pueden tener orientaciones bien definidas o no, de acuerdo con su arreglo geométrico.

Otra característica importante del comportamiento mecánico de la mampostería es su alta resistencia en compresión, comparada con la de tensión. En principio, este hecho no representa una dificultad desde el punto de vista del modelado numérico. Sin embargo, la fuerte asimetría del comportamiento en compresión y tensión de la mampostería, producto de su comportamiento no lineal, impide el uso de técnicas de análisis y evaluación sísmica desarrolladas para estructuras modernas, como pueden ser los análisis modales espectrales y, en general, cualquier técnica basada en la superposición. Por esta razón, una de las estrategias más usadas para evaluar la seguridad sísmica de construcciones históricas es la simulación del comportamiento de un modelo numérico sujeto a cargas laterales. Estas cargas se incrementan monótonamente hasta la falla, y el valor máximo se compara con la demanda sísmica esperada.

En los últimos años y en diversos países, el interés por conservar el patrimonio construido ante la amenaza de los sismos, ha llevado a diferentes grupos de investigación al desarrollo de una cantidad apreciable de modelos de análisis estructural para construcciones históricas de mampostería (Lemos, 1998; Lourenço, 2002). Se han propuesto diferentes modelos constitutivos no lineales, desde los muy simples hasta los más complejos, que pueden simular el comportamiento del material ante cargas cíclicas o monótonas. Se han utilizado; asimismo, diversos modelos estructurales. La elección de los modelos constitutivo y estructural, depende del grado de precisión buscado en el análisis para cada caso en particular.

La participación de los ingenieros en proyectos de conservación o restauración de edificios dañados por sismo tiene dos fases. Primero, es necesario evaluar la seguridad sísmica de la construcción y segundo, en caso de ser necesario, diseñar las soluciones de refuerzo. En ambas fases el ingeniero necesita herramientas de análisis adecuadas $\mathrm{y}$, posiblemente, diferentes entre sí. Para la evaluación sísmica es deseable el uso de una herramienta de análisis precisa, que permita tener una idea clara del comportamiento sísmico de la estructura, identificar los mecanismos de falla críticos y decidir acerca de la necesidad o no de refuerzo. Por otro lado, para el diseño del refuerzo, y dado el carácter iterativo del proceso, es deseable contar con herramientas simplificadas que permitan evaluar rápidamente distintas opciones.

Desde el punto de vista de la ingeniería estructural, es recomendable usar diferentes técnicas de análisis para monumentos que para centros históricos. Un monumento conjunta en una sola unidad todo su valor histórico y cultural, así como su comportamiento mecánico. Es de fundamental importancia que la evaluación sísmica sea tan precisa como sea posible y que las campañas de caracterización de materiales sean extensivas. Por el contrario, los centros históricos tienen su valor histórico y cultural disperso en múltiples construcciones, normalmente pequeñas. Las características mecánicas de los materiales 
presentan grandes variaciones de un edificio a otro, y es prácticamente imposible hacer una evaluación sísmica de cada unidad. En este caso, únicamente se puede estudiar un número limitado de edificios, seleccionados como críticos o representativos. Es común recurrir a herramientas de análisis más prácticas aunque, posiblemente, menos precisas. Para reducir las incertidumbres derivadas de la elección de los edificios estudiados y de la herramienta de cálculo, se recurre a los análisis paramétricos, que proporcionan una idea general de la importancia de cada parámetro utilizado en los análisis.

Con la finalidad de contribuir en la toma de decisiones acerca de la herramienta de análisis más adecuada para diferentes casos, en este artículo se comparan tres procedimientos de cálculo que se han desarrollado para el estudio de estructuras históricas. El Método de los Elementos Finitos (MEF) no lineal, El Método de los Elementos Rígidos (MER) y el Análisis Límite con Bloques Rígidos (ALBR). El primero es una herramienta que permite llegar a niveles de precisión bastante altos, si se utiliza adecuadamente, y si se conocen bien los parámetros de los materiales. El MEF es adecuado para el estudio de monumentos y estructuras grandes o complejas, pero que consume una gran cantidad de recursos computacionales y de tiempo de pre- y pos-procesamiento. En el MEF no lineal, como se presenta en este artículo, se utiliza un modelo bastante detallado del comportamiento del material, y se recurre al macromodelado para representar a la estructura. El (MER) recurre a un modelo no lineal del material menos detallado, y a un modelo simplificado de la estructura, estas simplificaciones lo hacen menos preciso, pero tienen la ventaja de consumir menos recursos que el MEF y de reducir los tiempos de pre y posprocesamiento. El MER es adecuado para el análisis de monumentos y edificios importantes, si se cuenta con recursos limitados, o para estructuras pequeñas. El ALBR es una herramienta simplificada tanto en términos del modelo constitutivo del material como del modelo estructural, pero que conserva los aspectos fundamentales del comportamiento mecánico de los edificios históricos de mampostería. Consume pocos recursos; sin embargo, proporciona resultados adecuados para estructuras relativamente pequeñas o simples. El ALBR es adecuado para análisis en centros históricos o para estimar cantidades de refuerzo en cualquier estructura cuando se conocen con claridad los modos de falla críticos. Estas herramientas, aunque reducidas en número, cubren un espectro amplio de precisión en el análisis de estructuras históricas de mampostería.

El objetivo principal de este artículo es presentar y comparar las herramientas de cálculo mencionadas, y a cuyo desarrollo han contribuido tres investigadores jóvenes mexicanos. Debido a limitaciones de espacio, a que los pormenores técnicos de cada herramienta ya se han publicado en otros foros, y pueden consultarse en las referencias, no se incluyen aquí discusiones acerca de las incertidumbres que implican los parámetros introducidos o descripciones detalladas de la forma de obtenerlos. En las referencias que se citan, se presentan cuestiones teóricas y técnicas acerca de los métodos en forma más minuciosa, así como con discusiones acerca de distintos aspectos involucrados en su precisión y confiabilidad. Este artículo presenta brevemente las herramientas de cálculo, las compara entre sí, al mismo tiempo que las valida mediante un ejemplo que representa el comportamiento sísmico típico de construcciones históricas de mampostería.

En la siguiente sección se mencionan brevemente las bases de cada método, los detalles no están dentro de los alcances de este trabajo y pueden consultarse en las referencias que se citan. A continuación, con fines de validación y de comparación, se presentan los resultados que se obtienen de aplicar estas técnicas de análisis a la simulación numérica de un modelo experimental de una subestructura de mampostería, que fue sujeta a cargas laterales para representar una acción sísmica equivalente. Los modelos corresponden con una arcada del claustro del Monasterio de San Vicente de Fora (Lisboa, Portugal), ensayado experimentalmente en el laboratorio ELSA, del Centro de Investigación Conjunta de la Comisión Europea (Ambrosetti, 1998; Pegon et al., 2001, Giordano et al., 2002). Finalmente, se incluyen las conclusiones pertinentes. 


\section{MODELOS DE ANÁLISIS}

Para fines de análisis estructural, el ingeniero necesita desarrollar modelos del comportamiento mecánico de los materiales, así como modelos estructurales de las construcciones. Los modelos de comportamiento mecánico de los materiales pueden variar ampliamente, desde los muy complejos y detallados, hasta los muy simples y aproximados. Los modelos detallados permiten predecir aproximadamente el comportamiento de las estructuras, siempre y cuando los parámetros del modelo, las condiciones de frontera de la estructura y las cargas se conozcan con buena precisión. Estos modelos pueden representar todas las características esenciales del comportamiento estructural y también muchas que pueden no ser fundamentales en la práctica. Los modelos detallados son adecuados en la evaluación de monumentos, por ejemplo, en donde es posible realizar campañas extensivas de caracterización de los materiales. En el otro extremo, los modelos de material muy simplificados producen información limitada y aproximada acerca del comportamiento estructural. Sin embargo, esta información puede ser suficiente en cantidad y calidad para fines de ingeniería, cuando la información que se dispone acerca de las propiedades mecánicas del material, las condiciones de apoyo y las cargas es igualmente aproximada. Este es el caso, por ejemplo, de edificios de mampostería en centros históricos cuando se evalúa la vulnerabilidad sísmica en conjunto.

Para elaborar el modelo de una estructura, ésta se divide en elementos. El comportamiento de cada elemento se representa en forma separada y, posteriormente, se ensambla el modelo de la estructura a partir de ellos. Hay también una gran variedad de tipos de elementos estructurales, desde los muy complejos hasta los muy simples. Los primeros permiten modelar prácticamente cualquier estructura; como ejemplo se tienen los elementos finitos continuos. En otros casos, el ingeniero suele tomar ventaja de características particulares de los elementos estructurales para introducir simplificaciones en el modelo, un ejemplo extensamente usado en estructuras modernas es el elemento viga. Una estrategia de modelado, que ha demostrado ser útil en estructuras de mampostería, es la combinación de elementos bloque, rígidos o deformables, con elementos interfaz. Este modelo es atractivo tanto para micro-modelos, en donde los elementos interfaz representan explícitamente a las juntas de mortero y a la interfaz entre mortero y pieza, mientras que los bloques representan a las piezas mismas; como para macro-modelos, en donde los elementos interfaz modelan las (posibles) grietas y los bloques representan porciones de mampostería sana, o poco dañada. El ALBR incorpora esta estrategia. Otra posibilidad es usar bloques rígidos que representan porciones de mampostería, unidos por interfaces cuyo comportamiento mecánico se obtiene mediante homogeneización de las propiedades del material, y que no representan explícitamente a las juntas. Este enfoque semi-continuo es el que se usa en el MER.

\section{Método de los elementos finitos}

En las dos últimas décadas se ha incrementado la implementación de modelos de comportamiento inelástico de materiales como la mampostería dentro del marco de los elementos finitos. Se pueden encontrar diversas formulaciones que logran simular la mecánica no-lineal de materiales casi-frágiles tales como los basados en agrietamiento distribuido (Rots, 1988), plasticidad donde se incorporan variables internas de ablandamiento, como los que presentan Feenstra (1993) y Lourenço (1996), modelos basados en daño del continuo (Simo y Ju, 1987), entre otros, que permiten observar la evolución del daño ante cargas estáticas o dinámicas de estructuras. Dentro de este contexto de modelos constitutivos, en este artículo se presenta un modelo de comportamiento del material basado en plasticidad que permite evaluar el daño en tensión en dos direcciones ortogonales, aplicada a macro-modelos de edifícios antiguos (Roeder, 2004). 
Los monumentos históricos en muchos casos requieren un tratamiento particular, porque así lo exigen la complejidad de sus geometrías, las condiciones de apoyo y el comportamiento inelástico de los materiales con los cuales se erigieron. En el caso de usar el MEF, las geometrías se definen con elementos finitos apropiados, según el grado de detalle que se necesite para capturar la información más relevante, y así estimar las condiciones de estabilidad en que se encuentran estas estructuras. El comportamiento inelástico del material, para el caso de la mampostería en construcciones históricas, está comúnmente asociado al daño que se produce por los esfuerzos de tensión. La evolución del daño se puede modelar asignando relaciones constitutivas adecuadas, que pueden deducirse a partir de las hipótesis que se emplean en teorías matemáticas tales como: la teoría de la plasticidad, modelos de daño del continuo, agrietamiento distribuido, entre otras, que limitan el espacio de esfuerzos posibles en el material.

Por ejemplo, se puede limitar el espacio de esfuerzos con una superficie de fluencia cuya expresión se describe en la ecuación 1. Este modelo de comportamiento, denominado modelo de Ranking ortótropo, está basado en la teoría de la plasticidad y usa dos variables internas, $\kappa_{1}$ y $\kappa_{2}$, las cuales evalúan las deformaciones relacionadas al daño en tensión del material en dos direcciones ortogonales. Este es el modelo que se emplea en el ejemplo más adelante. Los esfuerzos equivalentes $\bar{\sigma}_{1}$ y $\bar{\sigma}_{2}$ son funciones escalares prescritas que representan el comportamiento de ablandamiento del material en las direcciones $x$ e $y$, respectivamente. Estas funciones tienen como variables independientes a las deformaciones equivalentes $\kappa_{1}$ y $\kappa_{2}$ en las direcciones respectivas. $\sigma_{\mathrm{x}}$ y $\sigma_{\mathrm{y}}$ son los esfuerzos axiales en las direcciones $x \mathrm{e}$ $y$, respectivamente y $\tau_{\mathrm{xy}}$ es el esfuerzo cortante.

$$
f\left(\sigma, \kappa_{1}, \kappa_{2}\right)=\frac{\left(\sigma_{x}-\bar{\sigma}_{1}\left(\kappa_{1}\right)\right)+\left(\sigma_{y}-\bar{\sigma}_{2}\left(\kappa_{2}\right)\right)}{2}+\sqrt{\left(\frac{\left(\sigma_{x}-\bar{\sigma}_{1}\left(\kappa_{1}\right)\right)-\left(\sigma_{y}-\bar{\sigma}_{2}\left(\kappa_{2}\right)\right)}{2}\right)^{2}+\alpha \tau_{x y}^{2}}
$$

El parámetro $\alpha$ controla la contribución del esfuerzo cortante durante la evolución del daño en el material. Este valor se calcula con la ecuación 2.

$\alpha=\frac{f_{t x} \cdot f_{t y}}{\tau_{u}^{2}}$

En la ecuación 2, $f_{t x}$ y $f_{t y}$ representan los valores de las resistencias a tensión del material en las direcciones $x$ e $y$ respectivamente. El valor $\tau_{u}$ representa la resistencia al cortante del material.

Junto con el modelo de comportamiento y el tipo de elementos finitos escogido para representar la geometría de la estructura, es forzoso emplear más de un procedimiento de solución de las ecuaciones nolineales incrementales que se definen durante la evolución de las cargas estáticas o dinámicas. Esto último quiere decir que la matriz de rigidez tangente usada en la solución iterativa puede dejar de ser definida positiva al desarrollar el análisis, debido a la representación numérica del daño en los coeficientes de dicha matriz. Por esto se exige conocer los diversos procedimientos de solución basados en los métodos de Newton-Raphson para lograr una predicción adecuada de la respuesta global de la estructura ante la solicitación impuesta (Roeder, 2004).

\section{Método de los Elementos Rígidos}

Casolo $(2000,2004)$ primero, y Casolo y Peña $(2005,2007)$ después, desarrollaron el MER, un método de análisis simplificado que toma en cuenta las características inelásticas de la mampostería. En este método se discretiza la estructura de mampostería en elementos rígidos. Los elementos rígidos 
representan grandes porciones de mampostería y en los lados de conexión se concentran las deformaciones y los esfuerzos. El método toma en cuenta una ley histerética degradante, con la que se representa el comportamiento frágil de la mampostería. Esto conlleva a tener menos grados de libertad, en comparación con métodos más precisos, como el MEF no-lineal, por ejemplo, lo cual permite realizar análisis en menos tiempo, tanto computacional, como de preparación de datos e interpretación de resultados.

El MER introduce varias simplificaciones para el estudio estructural de construcciones de mampostería. En este método, las estructuras se consideran como un ensamble de elementos rígidos cuadriláteros. La cinemática de cuerpo rígido de los elementos incluye dos desplazamientos lineales y una rotación (figura 1). Los elementos rígidos se unen entre sí mediante tres puntos de conexión (resortes inelásticos), dos axiales, separados entre ellos para tomar en cuenta un par de fuerzas, mientras que el tercer punto es una unión de cortante, colocado al centro del lado de conexión (figura 2).

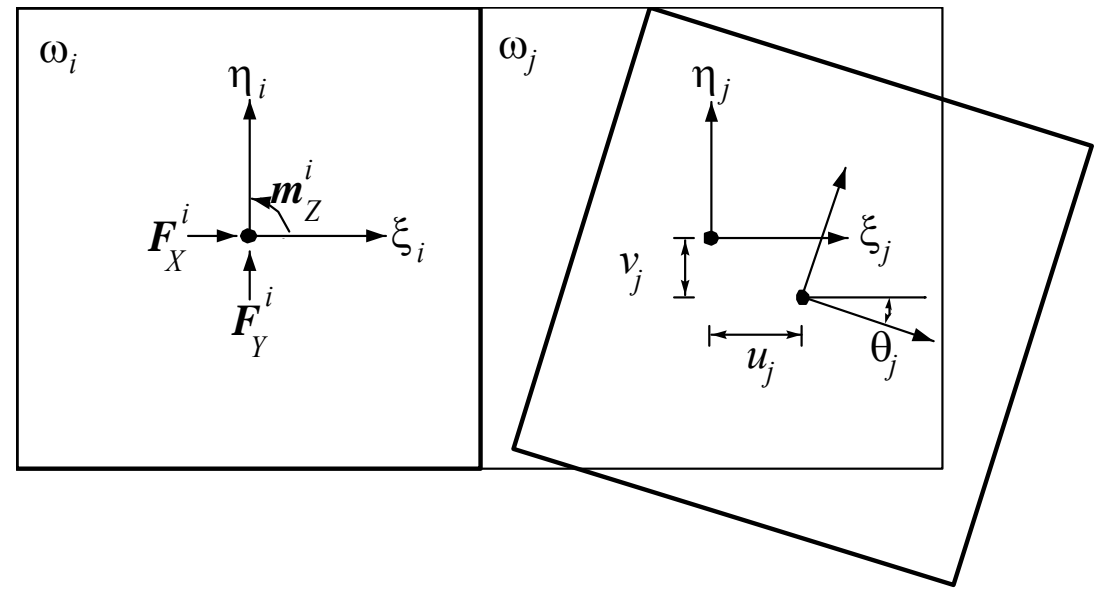

Figura 1. Fuerzas y desplazamientos de los elementos rígidos

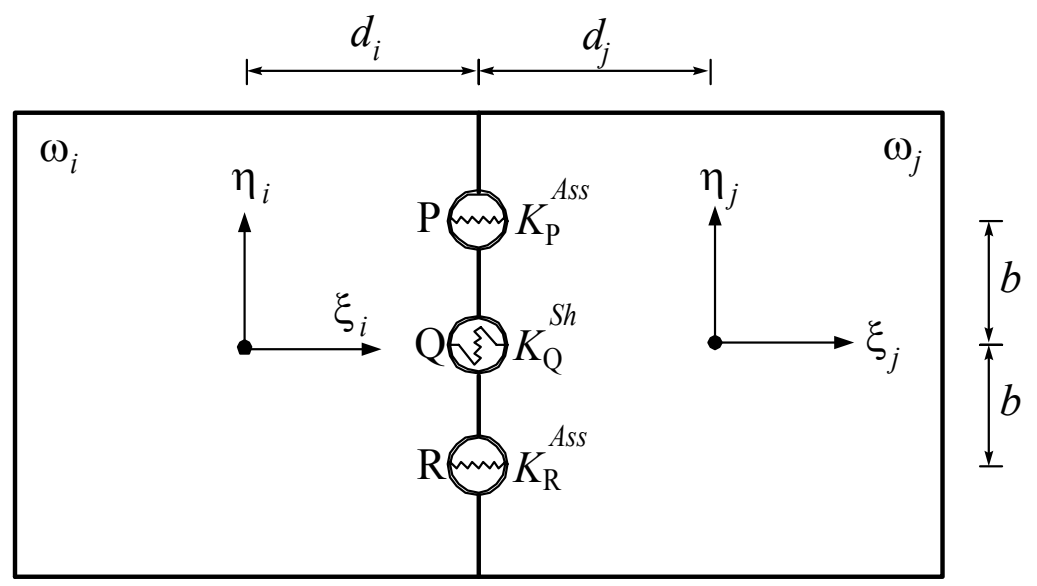

Figura 2. Conexión de dos elementos rígidos

En el planteamiento de este método, la mampostería se considera como un material deformable, pero esta deformación se concentra en los puntos de conexión, mientras que el elemento en sí es indeformable (rígido). Los puntos de conexión se encuentran cinemáticamente desacoplados entre sí; sin embargo, desde el punto de vista mecánico, se encuentran acoplados entre ellos mediante las leyes constitutivas del material. En otras palabras, los puntos de conexión representan las características 
mecánicas del material, pero al mismo tiempo representan la capacidad del modelo para tomar en cuenta la separación o deslizamiento entre elementos.

Un modelo de elementos rígidos puede ser considerado como semi-continuo. En un modelo continuo existe continuidad y compatibilidad entre los elementos que comparten un mismo nodo; mientras que en un modelo discontinuo, los elementos son capaces de separarse o deslizarse entre sí. En el MER puede existir un movimiento relativo entre dos elementos (separación o deslizamiento); sin embargo, las conectividades iniciales del modelo no cambian durante el análisis, y existe una continuidad relativa. De hecho, pueden presentarse separación, deslizamiento o superposición entre dos elementos adyacentes, que numéricamente significan tensión, cortante y compresión en los puntos de conexión, respectivamente. Se supone que cada elemento es independiente en su movimiento, ya que las estructuras de mampostería no pueden ser consideradas continuas, debido a que parte de su deformación se deriva de un movimiento relativo entre elementos; sin embargo, sigue existiendo una relación entre los esfuerzos y las deformaciones. Las conectividades iniciales no cambian durante el análisis para optimizar el tiempo de cómputo.

\section{Análisis límite con bloques rígidos}

Para fines de evaluación sísmica de construcciones históricas, a menudo es suficiente conocer las capacidades de carga lateral y los mecanismos de falla para direcciones preestablecidas del movimiento telúrico. Un modelo de material rígido-perfectamente plástico, dentro del marco del análisis límite, es capaz de proporcionar estos resultados. El atractivo de este modelo de material es que requiere solamente parámetros de resistencia, no es necesario evaluar ni rigidez ni parámetros de ablandamiento. En contrapartida, es imposible estimar las deformaciones de la estructura. Los resultados se reducen a la carga última, mecanismo de colapso y esfuerzos en los puntos o secciones críticos. Esta información es suficiente para la evaluación sísmica de edificios pequeños o de geometría simple. Asimismo, el ALBR es útil para diseño de refuerzo en construcciones mayores en tamaño o importancia, en las que se han identificado los mecanismos de falla mediante herramientas de análisis más precisas (Lourenço y Orduña, 2000).

El ALBR se basa en suponer que la estructura se puede modelar como un conjunto de bloques infinitamente rígidos que interactúan entre sí mediante interfaces. Cada bloque tiene asociados tres grados de libertad, dos traslaciones y una rotación, igual que en el MER (figura 1). Las interfaces son incapaces de soportar esfuerzos de tensión, pero pueden transmitir esfuerzos cortantes, limitados por el criterio de Coulomb sin cohesión, y esfuerzos de compresión. La formulación matemática consiste en buscar una solución que cumpla con el equilibrio, la compatibilidad, las condiciones de falla y las leyes de flujo. Mediante un proceso de solución propuesto por Orduña y Lourenço (2005b), se sigue aproximadamente la historia de carga lateral sobre la estructura al hacer variar el esfuerzo efectivo de compresión desde un valor muy pequeño (apenas el necesario para soportar las cargas permanentes) hasta su valor estimado.

Los antecedentes de este método se remontan a los siglos XVII y XVIII, cuando renombrados pensadores como Hooke, Coulomb o Poleni usaron conceptos, que hoy se pueden reinterpretar dentro de la teoría moderna del análisis límite, para la evaluación de la seguridad de construcciones de mampostería. En los primeros tiempos del desarrollo de la teoría del análisis límite, Kooharian (1952) y Heyman (1966, 1967 y 1969) establecieron las bases teóricas para el uso de esta herramienta en estructuras de mampostería. Livesley (1978 y 1992) y Gilbert y Melbourne (1994) propusieron formulaciones del ALBR como problemas de programación lineal. Begg y Fishwick (1995) y Baggio y Trovalusci (1998) abordaron el problema del ALBR con leyes de flujo no asociadas en donde el factor de carga límite no es único. Orduña y Lourenço (2005a y b) propusieron un proceso de solución que sigue aproximadamente la 
historia de carga, para encontrar el factor de carga límite y el mecanismo de falla para problemas de ALBR con flujo no asociado.

\section{MODELOS DEL MONASTERIO DE SAN VICENTE DE FORA}

El monasterio de San Vicente de Fora, ejemplo de la arquitectura portuguesa del siglo XVI, representa el monumento histórico típico de la ciudad de Lisboa. La estructura resistente está conformada por columnas y arcos de bloques de piedra unidos con mortero, en combinación de muros de relleno de mampostería (figura 3). Este monumento sobrevivió al gran sismo de 1755 que devastó Lisboa; sin embargo, presenta signos aún visibles de los efectos de dicha catástrofe.

Como parte de un programa de investigación, cuyos objetivos fueron la estimación de la vulnerabilidad sísmica del monasterio y la evaluación de soluciones y técnicas de refuerzo de monumentos históricos, en el laboratorio ELSA, del Centro de Investigación Conjunta de la Comisión Europea, se efectuaron varias pruebas en un modelo a escala natural de una sección de la fachada interior que se muestra en la figura 3 (Ambrosetti, 1998; Pegon et al., 2001, Giordano et al., 2002). La figura 4 presenta la geometría del modelo experimental y la figura 5 muestra esquemáticamente la configuración de los actuadores para cargas verticales y horizontal.

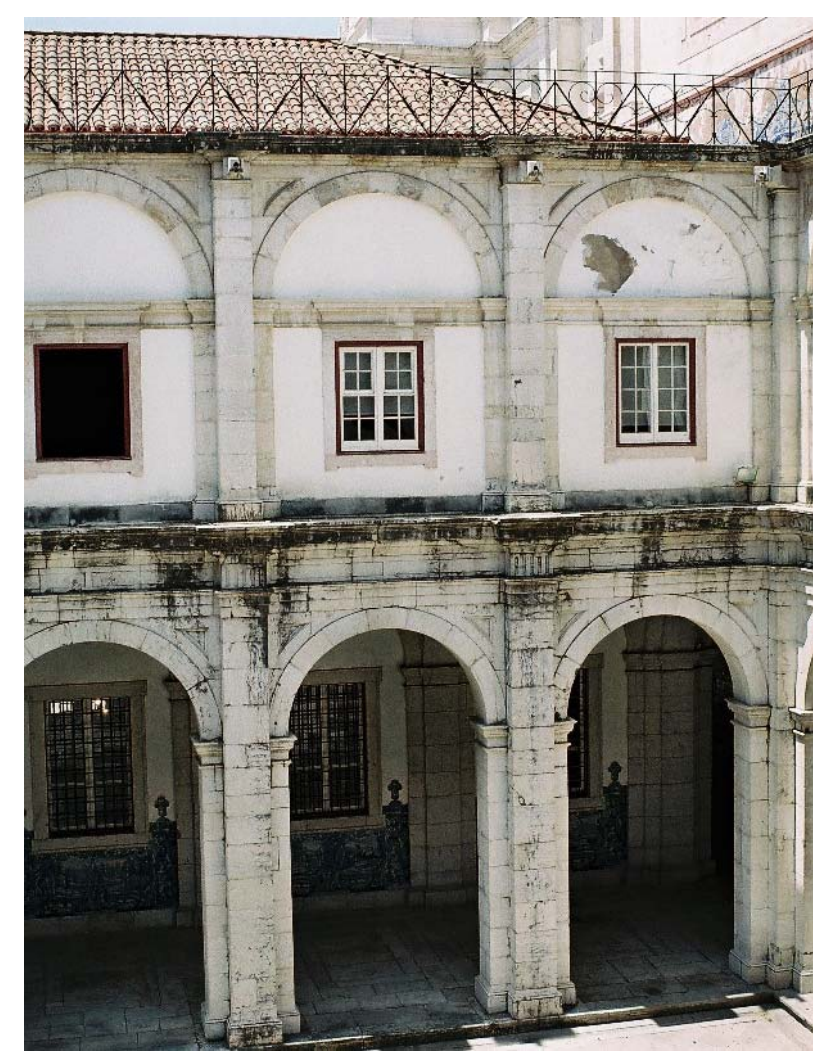

Figura 3. Detalle de la arcada del Monasterio de San Vicente de Fora 


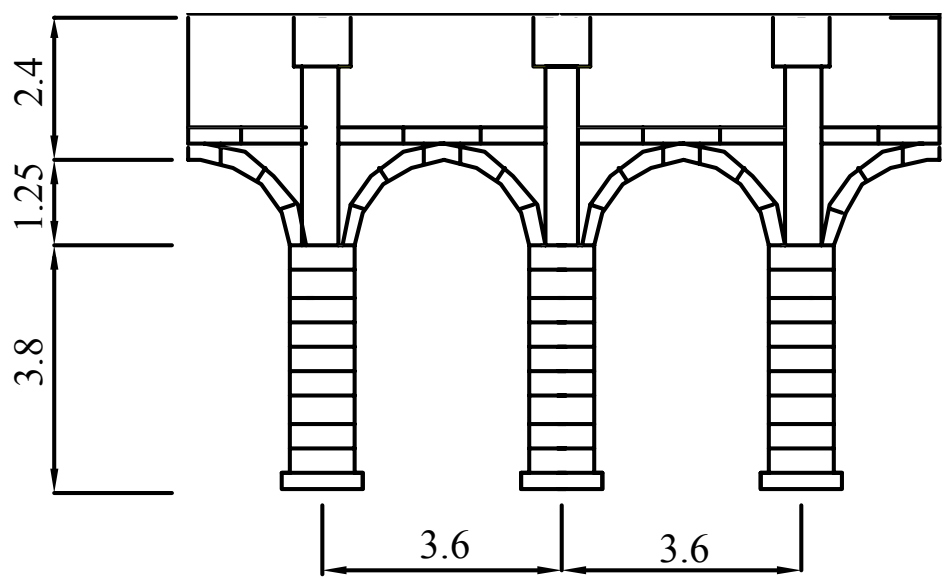

Figura 4. Geometría del modelo ensayado (dimensiones en $\mathrm{m}$ )

El espécimen ensayado consta de un arreglo ordenado de bloques de piedra unidos con mortero en columnas y arcos, así como de mampostería de relleno. El arreglo de bloques y las propiedades del material utilizado en el prototipo, reproducen los de la estructura real. Con el fin de simular las condiciones del sub-ensamble dentro de la estructura completa, la parte superior del modelo fue confinada horizontalmente mediante barras pos-tensadas, que proporcionaron una carga de $350 \mathrm{kN}$. La presencia de pisos superiores se tomó en cuenta mediante la aplicación de cargas verticales de $400 \mathrm{kN}$ en la parte superior de cada columna y de $100 \mathrm{kN}$ en cada panel de mampostería de relleno. Asimismo, se restringieron los desplazamientos verticales en la parte superior de las dos columnas externas. Las acciones sísmicas sobre el modelo se consideraron mediante la aplicación de fuerzas horizontales igualmente distribuidas en la parte superior de las tres columnas a través de un marco de carga. El programa de pruebas consistió en ensayes tanto seudo-dinámicos, como casi-estáticos cíclicos (Ambrosetti, 1998).

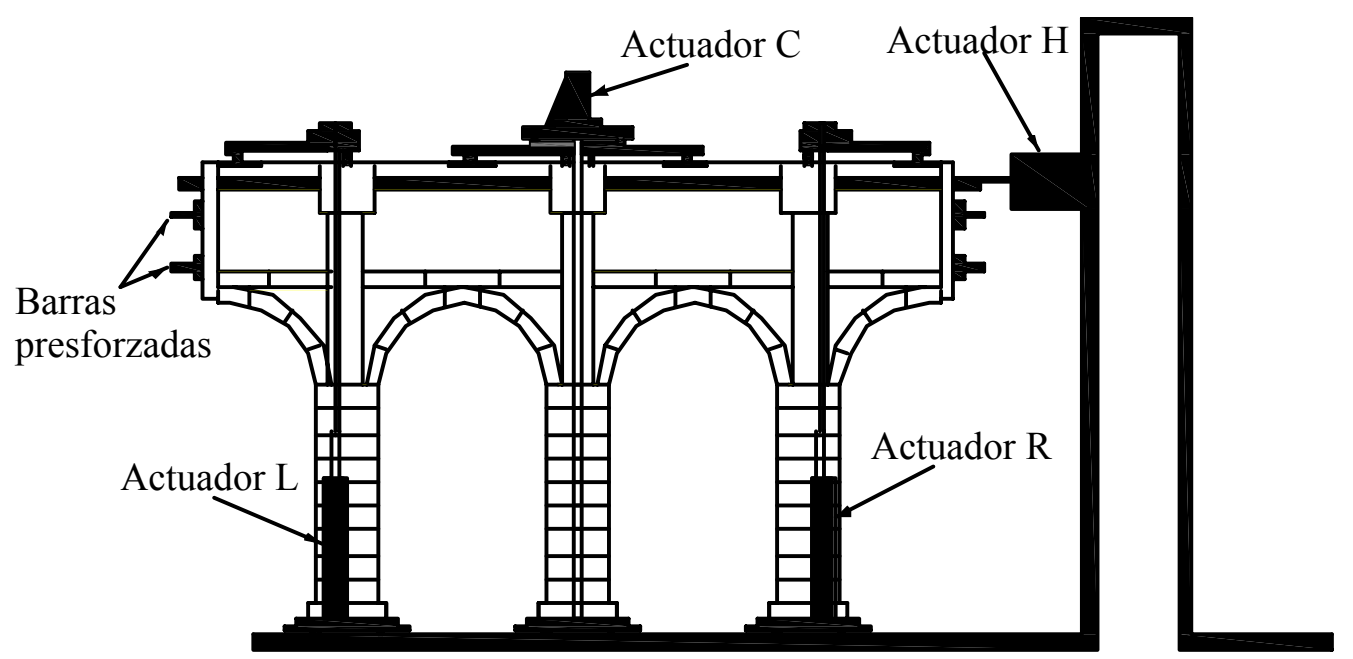

Figura 5. Modelo utilizado en el Laboratorio ELSA (Ambrosetti, 1998)

Los valores del módulo de Young $(E)$, el coeficiente de Poisson $(v)$, la densidad $(\rho)$, las resistencias a la tensión $\left(\sigma_{\mathrm{t}}\right)$ y a compresión $\left(\sigma_{\mathrm{c}}\right)$ de los materiales que se utilizaron en los modelos numéricos de la estructura se basan en los medidos en el experimento de acuerdo con la tabla 1. El valor de resistencia a la tensión del mortero considera que el material, en general, tiene un desempeño pobre bajo esta acción. 
Tabla 1. Parámetros de los materiales (Ambrosetti, 1998; Pegon et al., 2001)

\begin{tabular}{cccccc}
\hline Material & $\begin{array}{c}E \\
(\mathrm{GPa})\end{array}$ & $v$ & $\begin{array}{c}\rho \\
\left(\mathrm{kg} / \mathrm{m}^{3}\right)\end{array}$ & $\begin{array}{c}\sigma_{\mathrm{t}} \\
(\mathrm{Mpa})\end{array}$ & $\begin{array}{c}\sigma_{\mathrm{c}} \\
(\mathrm{MPa})\end{array}$ \\
\hline Bloques & 110.0 & 0.2 & 2600 & 6.60 & 66.77 \\
Mampostería & 1.0 & 0.2 & 1500 & 2.98 & 13.52 \\
Junta & 2.5 & 0.2 & 1800 & 2.65 & 23.10 \\
\hline
\end{tabular}

\section{Modelo de elemento finitos}

El modelo de elementos finitos que se presenta a continuación se basa en los presentados por Guzmán et al. (2001). Roeder (2004) presenta algunos experimentos numéricos para proponer el valor de la energía de fractura que garantice la estabilidad de proceso de solución y con base en sus resultados propone un valor de $0.02 \mathrm{~N}-\mathrm{mm} / \mathrm{mm}^{2}$ en ambas direcciones materiales, $x$ e $y$. Este valor de energía de fractura se ajusta al tamaño de los elementos finitos que se utilizaron en el modelo numérico (figura 6) dividiendo dicha energía entre una dimensión representativa de la geometría del elemento; por ejemplo, la longitud de la diagonal de los elementos rectangulares o la altura promedio de los elementos triangulares. El modelo tiene 1,284 elementos triangulares de tres nodos y 5,238 elementos cuadriláteros de cuatro nodos, para un total de 6,522 elementos. Tiene; asimismo, alrededor de 30,700 grados de libertad. La función de esfuerzos-deformaciones equivalentes, que simula el ablandamiento del material, se supone lineal. El modelo de plasticidad incluye un parámetro $\alpha$, que se obtiene de la división del producto de las resistencias a tensión en las direcciones $x$ e $y$, entre la resistencia al cortante del material elevada al cuadrado. Lourenço (1998) demuestra que el parámetro $\alpha$, cuyos valores normalmente están entre $1.0 \mathrm{y}$ 2.0, puede introducir variaciones importantes en la respuesta no-lineal a tensión de los modelos numéricos de mampostería. Roeder (2004) hace una calibración de este parámetro comparando resultados experimentales con resultados obtenidos del análisis numérico. En este trabajo se observa que la resistencia en el modelo numérico se incrementa al aumentar el valor de $\alpha$. Por ejemplo, en los análisis de esta estructura y para un desplazamiento de $8.75 \mathrm{~mm}$, usando un valor de $\alpha=1.781$, la resistencia en el modelo numérico era de $547.23 \mathrm{kN}$. En otro caso, empleando un valor de $\alpha=1.324$, se obtiene una resistencia de $347.34 \mathrm{kN}$. Los resultados de Roeder (2004) indican que $\alpha=1.5$ es apropiado para este modelo en particular, con este valor la resistencia que se obtiene para el mismo desplazamiento es de $484.21 \mathrm{kN}$. Es necesario indicar que, en un caso práctico en el que se tenga control de la caracterización del material, es fundamental emplear resistencias de tensión en ambas direcciones materiales que se obtengan de ensayes en especimenes simples de mampostería que estén sometidas a trayectorias de carga similares al modelo numérico final. De esta manera se evitaría la necesidad de hacer estudios paramétricos para conocer la influencia de $\alpha$ en la respuesta del modelo.

\section{Modelo de elementos rígidos}

La figura 7 muestra la malla de elementos rígidos con la que se modeló la estructura. Se utilizaron 198 elementos rígidos con 584 grados de libertad en total. El material se consideró isótropo con las características individuales indicadas en la tabla 1. Los bloques y las juntas se consideraron como un material homogéneo, ya que los elementos rígidos son más grandes que las piezas del modelo físico. Para calcular las propiedades mecánicas, se realizó un procedimiento de homogeneización de los materiales del mortero y las piezas (Peña, 2001), que dio por resultado los siguientes parámetros: $E=10.5 \mathrm{GPa} ; v=0.2$; $\rho=2,600 \mathrm{~kg} / \mathrm{m}^{3} ; \sigma_{\mathrm{c}}=30.75 \mathrm{MPa} ; \sigma_{\mathrm{t}}=2.65 \mathrm{MPa}$. 


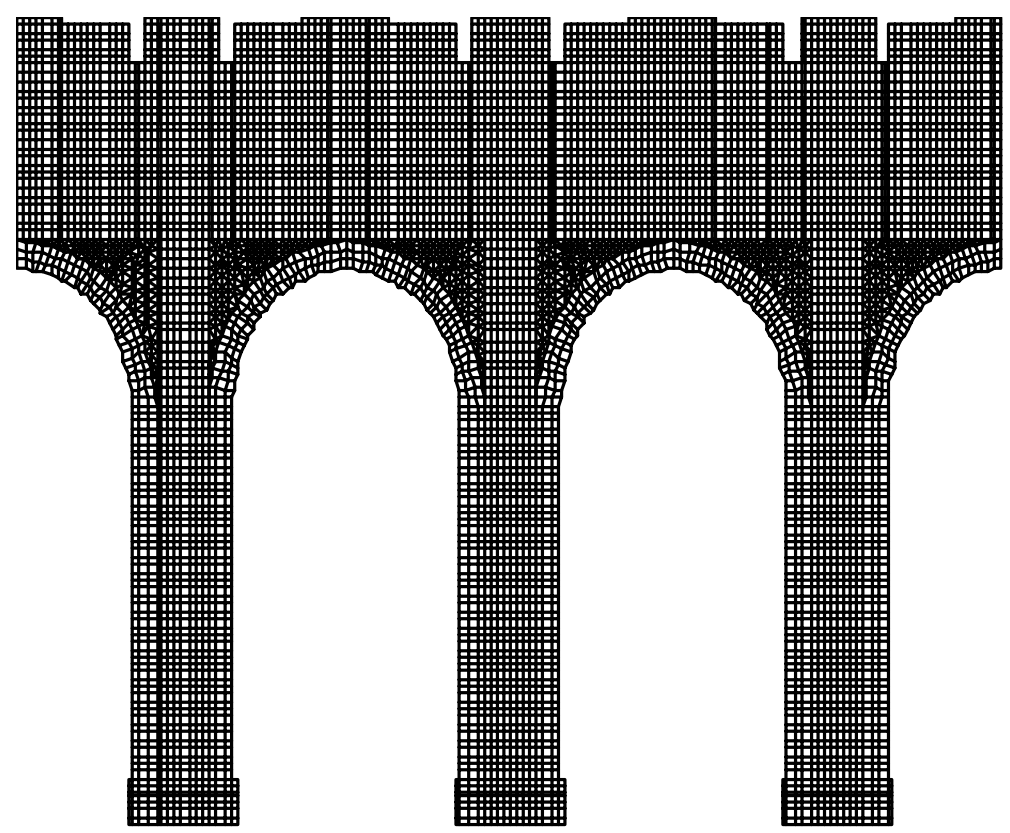

Figura 6. Modelo de elementos finitos de la estructura

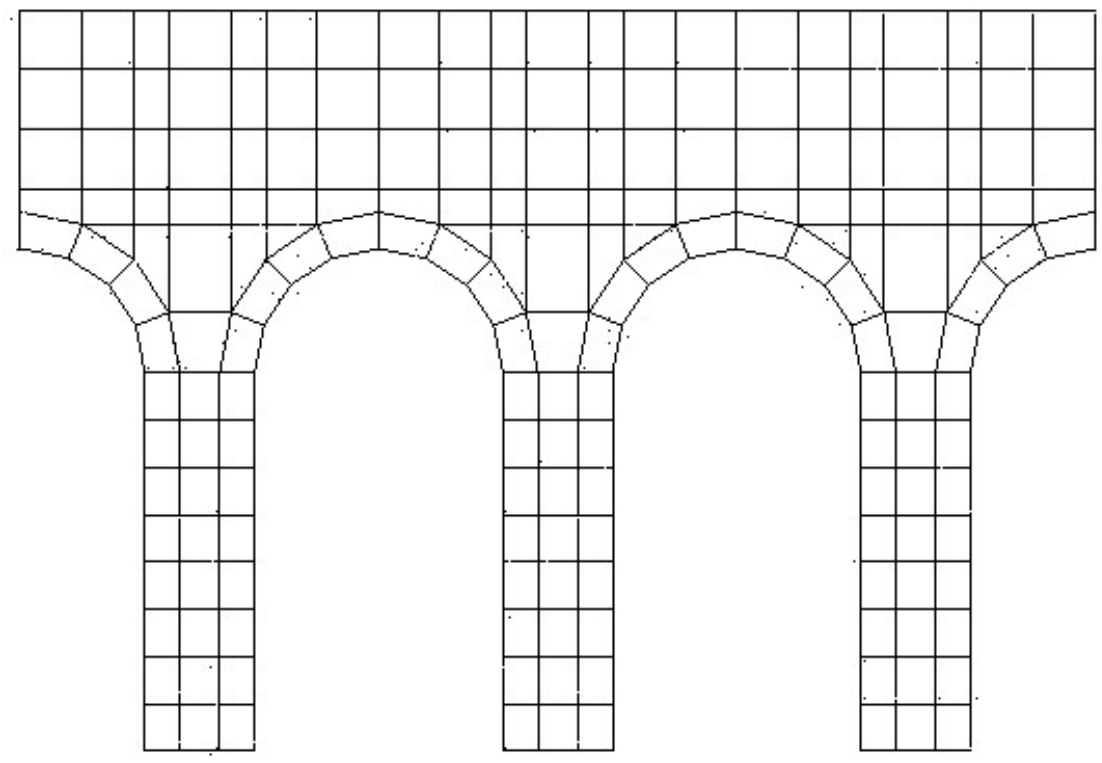

Figura 7. Modelo de elementos rígidos

\section{Modelo de análisis límite}

La figura 8 muestra el modelo de que se usó para el ALBR de la estructura en estudio. El modelo tiene 190 bloques rígidos, que corresponden con 570 grados de libertad; asimismo, tiene 444 elementos junta y 2 elementos barra. Este análisis está basado en la formulación presentada en Orduña y Lourenço (2003), con el procedimiento de solución propuesto en Orduña y Lourenço (2005b). En esta formulación es posible limitar los esfuerzos de compresión a un valor predeterminado, llamado esfuerzo efectivo, que normalmente es una fracción de la resistencia uniaxial y que toma en cuenta el posible 
agrietamiento transversal y la reducida ductilidad del material. Para este caso se tomó un esfuerzo efectivo de $8.55 \mathrm{MPa}$, con base en la información de la tabla 1 y las fórmulas presentadas en Nielsen (1999).

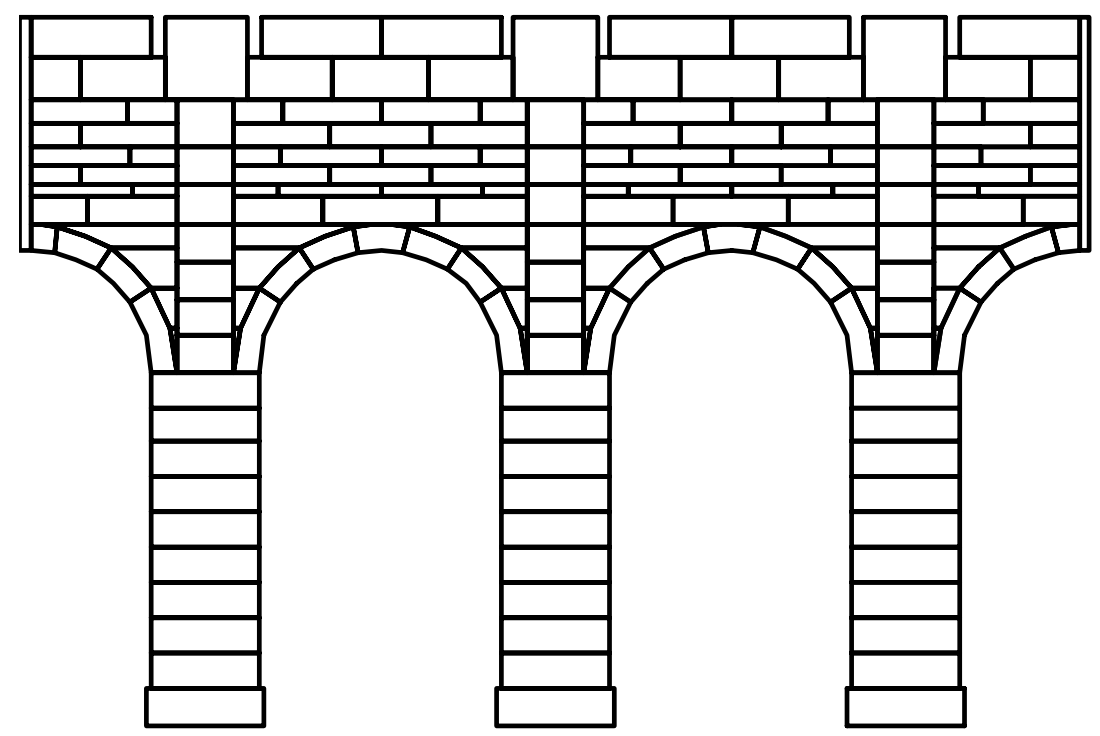

Figura 8. Modelo de bloques rígidos

\section{RESULTADOS}

La figura 9 muestra la curva fuerza-desplazamiento para los modelos numéricos junto con la obtenida en el ensayo experimental. Tanto la fuerza como el desplazamiento corresponden con las componentes horizontales medidas a la altura del actuador. En esta gráfica el resultado del ALBR se representa con una recta horizontal puesto que este método no estima desplazamientos. Se puede observar que, cualitativamente, las curvas de los modelos numéricos del MEF y el MER, son similares a la del experimento. Cuantitativamente, el modelo del MEF presenta una resistencia mayor en la parte final del experimento. Para un desplazamiento de $30 \mathrm{~mm}$, el experimento indica una carga lateral de $502 \mathrm{kN}$. Para ese mismo desplazamiento, el modelo del MEF predice una carga de $561 \mathrm{kN}$, que resulta ser $11 \%$ mayor que la del experimento. El modelo del MER presenta una resistencia ligeramente menor que la experimental. El modelo del MER predice una carga máxima de $466 \mathrm{kN}, 7 \%$ inferior que la experimental. Mientras que el modelo de ALBR predice más cercanamente la resistencia del experimento. Del ALBR resulta una resistencia de $509 \mathrm{kN}$, únicamente $1 \%$ superior a la experimental.

Las distribuciones de esfuerzos principales obtenidos con el MEF se muestran en la figura 10. En rojo se representan los esfuerzos principales mayores (tensión) y en azul los menores (compresión). La figura 10a muestra los resultados obtenidos del análisis para una fuerza lateral de aproximadamente $200 \mathrm{kN}$ y un desplazamiento de $0.09 \mathrm{~mm}$, que corresponde con un estado previo al inicio del agrietamiento en el modelo numérico. La máxima magnitud de los esfuerzos principales a tensión que se alcanza es de $2.543 \mathrm{MPa}$ y el mayor valor de esfuerzo principal a compresión es de $3.941 \mathrm{MPa}$. La figura 10b presenta los resultados al final del análisis, para una carga de aproximadamente $587 \mathrm{kN}$ que corresponde con un desplazamiento de $38.2 \mathrm{~mm}$. Estos resultados muestran que en los últimos estados de carga gobierna el comportamiento a compresión. En este nivel de carga los valores de esfuerzos principales a tensión en las zonas de daño oscilan entre $1.754 \mathrm{MPa}$ y $2.332 \mathrm{MPa}$; los valores máximos en compresión están alrededor de 4.7 MPa. 


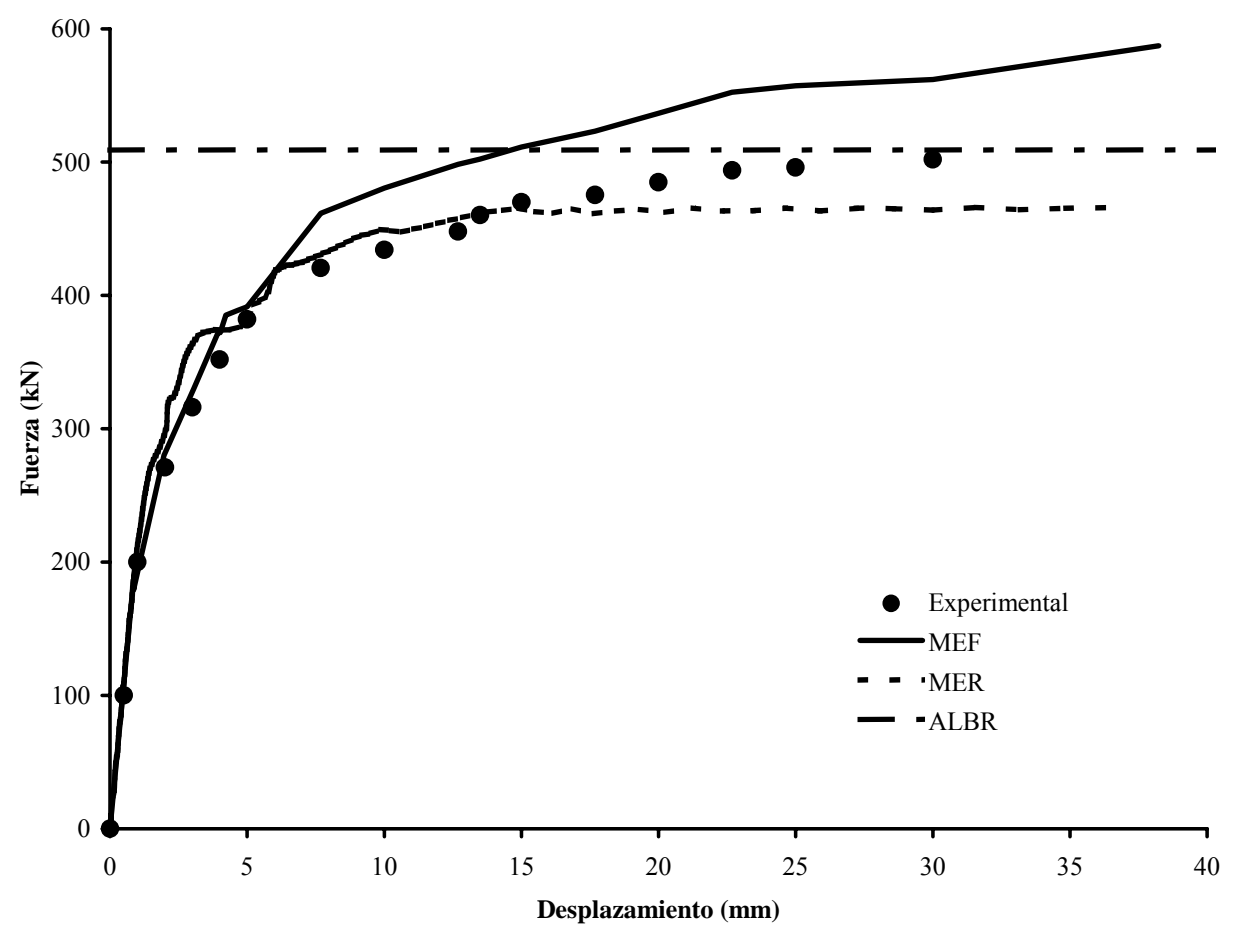

Figura 9. Curvas fuerza-desplazamiento

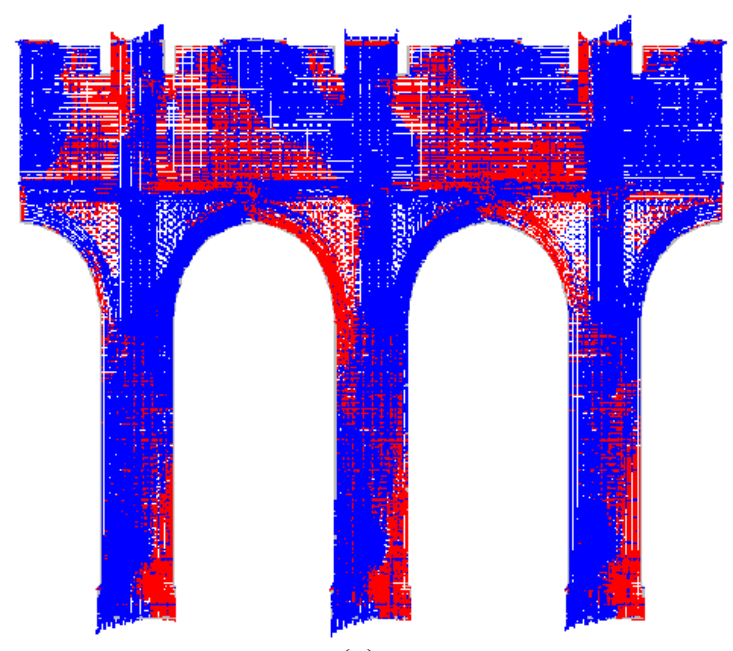

(a)

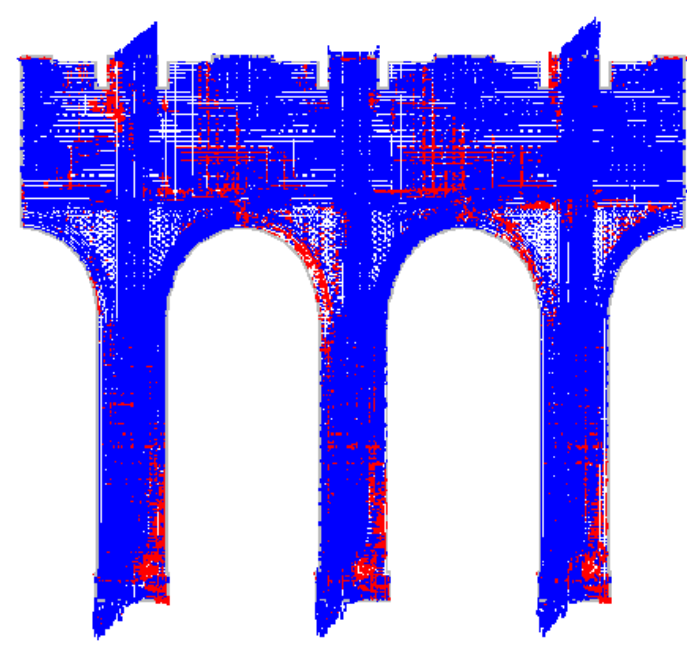

(b)

Figura 10. Esfuerzos principales; (a) antes del agrietamiento y (b) al final de la simulación numérica del MEF

La figura 11 presenta la distribución de esfuerzos axiales obtenidos al final del análisis con el MER. Se observa que, en el capitel y en la base de las columnas, los esfuerzos de compresión máximos se localizan en extremos opuestos, esto indica que la línea de presiones es inclinada. Los esfuerzos máximos de compresión son del orden de los $6 \mathrm{MPa}$, muy por debajo de la resistencia a compresión. Por tanto, la falla de la estructura se debe a la formación de un mecanismo y no al aplastamiento del material. Así 
mismo, se observa también una concentración de esfuerzos de tensión entre las dovelas del arco y el relleno de mampostería, que indica una separación entre estos materiales.

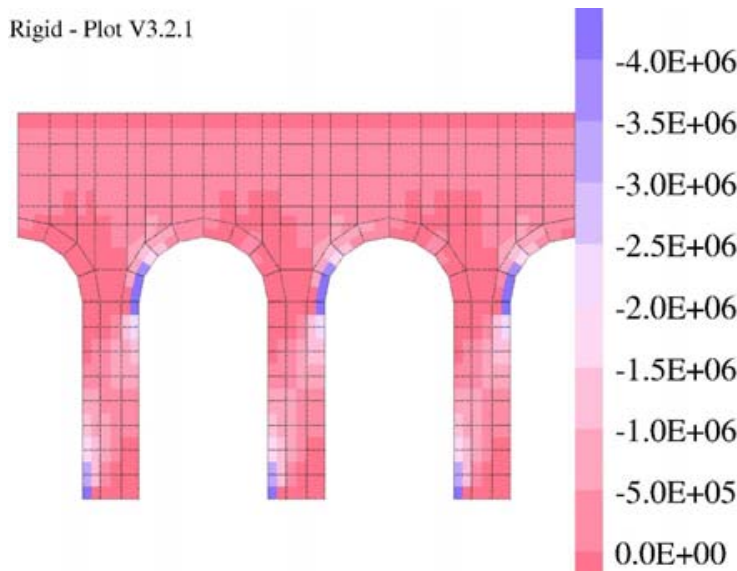

(a)
Rigid - Plot V3.2.1

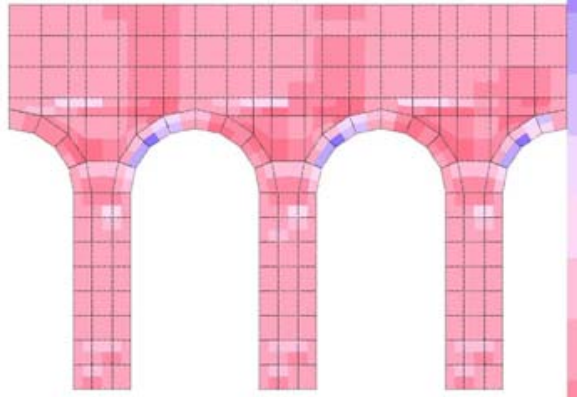

(b)
$-1.0 \mathrm{E}+06$

$-8.5 \mathrm{E}+05$

$-7.0 \mathrm{E}+05$

$-5.5 \mathrm{E}+05$

$-4.0 \mathrm{E}+05$

$-2.5 \mathrm{E}+05$

$-1.0 \mathrm{E}+05$

$5.0 \mathrm{E}+04$

$2.0 \mathrm{E}+05$

Figura 11. Esfuerzos axiales finales obtenidos con el MER (en Pascales); (a) verticales y (b) horizontales

La figura 12 presenta las fuerzas resultantes en las interfaces del modelo de ALBR. Aún cuando no es posible identificar un patrón definido de los empujes en la mampostería de relleno o en los arcos, se hace evidente con mucha claridad el empuje inclinado en las columnas.

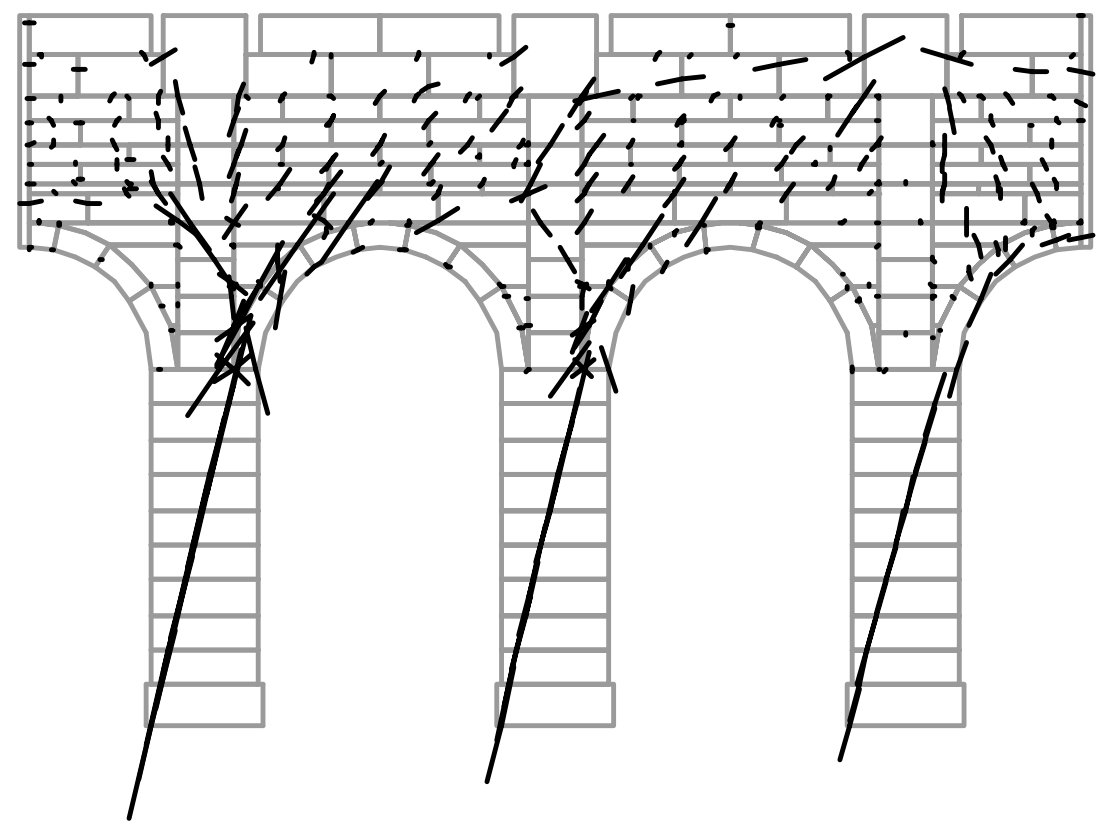

Figura 12. Líneas de empuje en las interfaces obtenidas del ALBR

Al comparar las figuras 10b, 11 y 12, se observa una clara congruencia en las distribuciones de esfuerzos de compresión en las columnas, evidenciando el empuje inclinado. En la figura 10a se observan esfuerzos principales de tensión entre los arcos y el relleno, en la parte izquierda de los arcos centrales y de las bases de las columnas. Estos esfuerzos han disminuido en la figura 10b debido al ablandamiento, e indicando agrietamiento en estas zonas. La figura 11 indica que el MER concuerda con esta distribución 
de tensiones y, en consecuencia, del agrietamiento. En el ALBR no existen esfuerzos de tensión en las interfaces, y no es posible extraer conclusiones de este tipo de la figura 12.

El patrón de agrietamiento en el modelo experimental se puede observar en la figura 13a. La figura 13b muestra las concentraciones de deformaciones plásticas principales obtenidas con el MEF. Estas deformaciones indican agrietamiento, por lo tanto la figura $13 \mathrm{~b}$ puede interpretarse como la localización del agrietamiento que resulta del análisis con el MEF. En la figura 13c se representa la distribución de daño a tensión de acuerdo con el análisis del MER. Las zonas en negro indican los puntos de conexión que han sobrepasado la resistencia a tensión del material, lo que físicamente representarían zonas de agrietamiento. El resultado del ALBR que indica la distribución del daño es el mecanismo de colapso y que, para el caso estudiado, se presenta en la figura 13d. Evidentemente, en esta figura, el agrietamiento está asociado con las juntas abiertas en el mecanismo de falla.

Puede observarse que el modelo experimental presenta agrietamiento entre los arcos y el relleno, al interior del relleno, principalmente entre éste y la continuación de las columnas, entre las dovelas de los arcos, y en la base de las columnas. La distribución de los esfuerzos principales de tensión obtenidos con el MEF, figura 10b, y la localización de agrietamientos, figura 13b, concuerdan bastante bien con el daño observado en el modelo experimental, figura 13a. Asimismo, coinciden con el experimento las zonas dañadas de acuerdo con el MER, figura 13c, y los desplazamientos relativos entre bloques obtenidos en el ALBR, figura 13d.

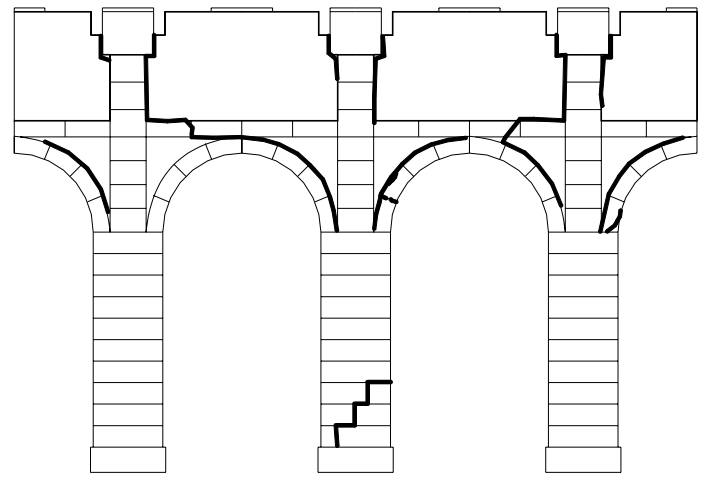

(a)

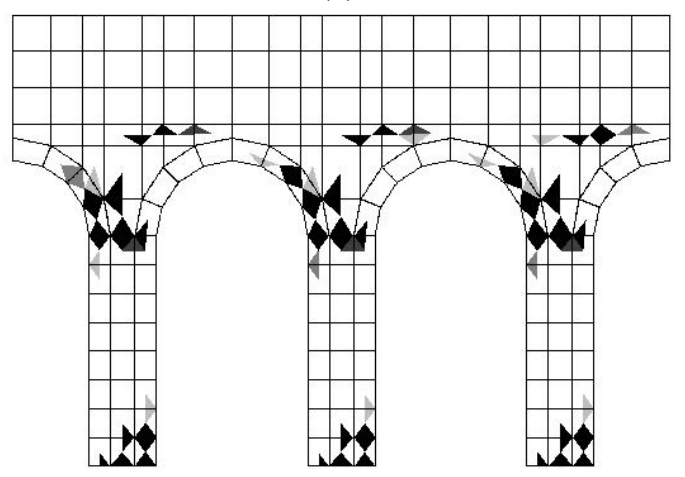

(c)

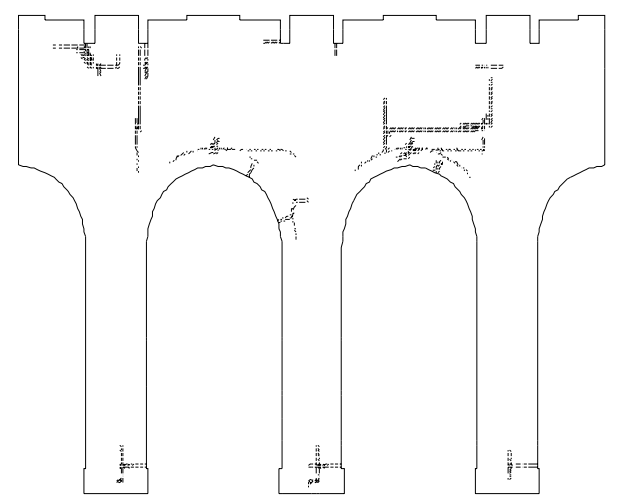

(b)

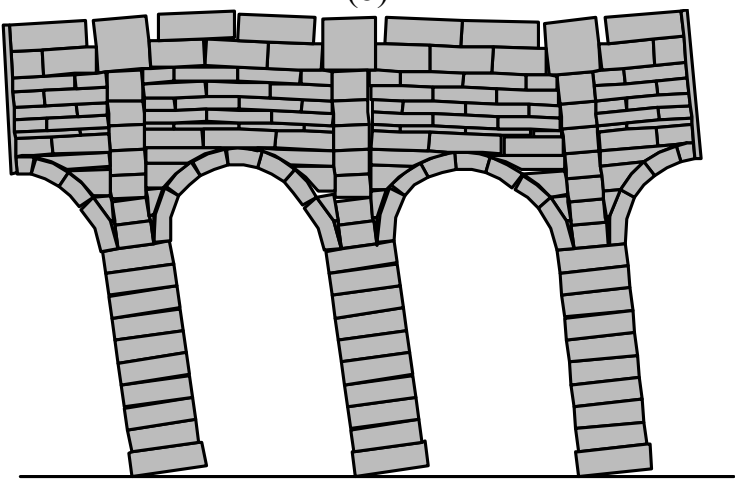

(d)

Figura 13. Patrones de agrietamiento; (a) agrietamiento en el modelo experimental; (b) MEF, deformaciones plásticas principales mayores; (c) MER, daño por tensión; y (d) ALBR, mecanismo de colapso 


\section{CONCLUSIONES}

Se aplicaron tres métodos diferentes al análisis numérico de una subestructura de un edificio histórico de mampostería sujeta a cargas laterales. Los tres métodos produjeron resultados adecuados, y congruentes con lo que se esperaba de ellos. El MEF permite emplear relaciones constitutivas no lineales complejas que simulan, con bastante precisión, la respuesta de las estructuras en ese intervalo. Es un método ampliamente usado en la evaluación sísmica de edificios históricos. Sin embargo, requiere de muchos parámetros, algunos de ellos, como $\alpha$, pueden ser difíciles de estimar en la práctica. Asimismo, este método requiere de una gran cantidad de tiempo en la elaboración del modelo, análisis e interpretación de resultados. El MER, es menos elaborado que el anterior, requiere de menos parámetros para caracterizar a los materiales, y produjo resultados igualmente adecuados. Finalmente, el ALBR, requiere un mínimo de parámetros, la realización de los modelos, del análisis y la interpretación de resultados son tareas bastante rápidas. La interpretación de resultados de este método es muy directa y visual, ya que las líneas de empuje y el mecanismo de colapso dan una idea bastante clara del comportamiento a la falla de la estructura. Los resultados de este análisis fueron también cercamos al comportamiento del modelo experimental. Por otro lado, tiene la desventaja de que no es posible estimar desplazamientos.

Los tres modelos de análisis produjeron buenos resultados para la subestructura considerada en este artículo. Sin embargo, este es un ejemplo pequeño y de comportamiento relativamente simple. Para estructuras más complejas, tanto arquitectónica como estructuralmente, debe esperarse que el MEF produzca resultados más precisos. Mientras que el MER y el ALBR, debido a sus hipótesis simplificadoras, pueden ser menos consistentes. Aún cuando sus resultados pueden ser suficientemente confiables, dentro de los límites de incertidumbre manejados normalmente en la ingeniería sísmica de edificios históricos. Esta conclusión se basa en la génesis de cada uno de los métodos; sin embargo, queda pendiente su verificación mediante la aplicación y comparación de los métodos a estructuras más grandes y complejas.

Como recomendación puede decirse que los métodos de análisis simplificados, tales como el ALBR o el MER, son adecuados para estudios preliminares, donde se requiere una evaluación rápida y confiable de la capacidad sísmica de construcciones antiguas. También son útiles cuando la estructura no es demasiado compleja, cuando la información es limitada, o cuando se diseñe una propuesta de reforzamiento. El MEF no-lineal tiene la capacidad de proporcionar resultados bastante más precisos, confiables y detallados, y se recomienda en la evaluación de la seguridad sísmica de estructuras complejas o muy importantes. Sin embargo, no debe olvidarse que la calidad de los resultados de un análisis estructural nunca será mejor que la de la información de partida. En este caso, el uso de modelos de material muy complejos trae como desventaja la necesidad de evaluar parámetros que son difíciles de obtener, y cuyo impacto en los resultados es necesario estimar mediante estudios paramétricos. Tampoco debe olvidarse que lo que se presentan aquí son herramientas de cálculo, que en ningún momento pueden sustituir el juicio y experiencia de un Ingeniero, quien es responsable de dar la justa interpretación a los resultados obtenidos de cualquiera de ellas.

\section{AGRADECIMIENTOS}

Los primeros dos autores agradecen el financiamiento de la Subsecretaría de Educación Superior de la SEP mediante recursos de los proyectos Promep/103.5/04/1322 y Promep/103.5/05/1880, respectivamente. Asimismo, se agradece el financiamiento parcial de la Universidad de Colima mediante el proyecto FRABA 362/05. 


\section{REFERENCIAS}

Ambrosetti, C (1998). "Sul comportamento sismico de un modulo strutturale di un monumento ad arco: Analisi numerica con modello ad attrito," Tesis de licenciatura, Politécnico de Milán, Milán, Italia, $157 \mathrm{pp}$.

Baggio, C y P Trovalusci (1998). "Limit analysis for no-tension and frictional three-dimensional discrete systems," Mechanics of Structures and Machines, Vol. 26, No. 3, pp. 287-304.

Begg, D W y R J Fishwick (1995). "Numerical analysis of rigid block structures including sliding," III International Symposium on Computational Methods in Structural Masonry, Lisboa, Portugal, J Middleton, y G N Pande (editores), pp. 177-183.

Casolo, S (2000). "Modelling the out-of-plane seismic behaviour of masonry walls by rigid elements," Earthquake Engineering and Structural Dynamics, Vol. 29, No. 12, pp. 1797-1813.

Casolo, S (2004). "Modelling in-plane microstructure of masonry walls by rigid elements," International Journal of Solids and Structures, Vol. 41, pp. 3625-641.

Casolo, S y F Peña (2005). "Modelo de elementos rígidos para el análisis de estructuras de mampostería". Revista Internacional de Métodos Numéricos para el Cálculo y Diseño en Ingeniería, Vol. 21, No. 2, pp. 193-211.

Casolo, S y Peña F (2007). "Rigid element model for in-plane dynamics of masonry walls considering hysteretic behaviour and damage," Earthquake Engineering and Structural Dynamics, Vol. 36, pp. 1029-1048

Feenstra, P H (1993). "Computational aspects of biaxial stress in plain and reinforced concrete", Tesis de doctorado, Universidad Técnica de Delft, Delft, Holanda.

Gilbert, M y C Melbourne (1994). "Rigid-block analysis of masonry structures," The Structural Engineer, Vol. 72, No. 21, pp. 356-361.

Giordano, A, E Mele y A De Luca (2002). "Modelling of historical masonry structures: comparison of different approach through a case study," Journal of Engineering Structures, Vol. 24, pp. 10571069.

Guzmán, M, G M Roeder y G Ayala (2002). "Modelado del comportamiento no lineal de una estructura histórica de mampostería mediante elementos finitos," XIII Congreso Nacional de Ingeniería Estructural, Puebla, México.

Heyman, J (1966). “The stone skeleton”, International Journal of Solids and Structures, Vol. 2, pp. 249279.

Heyman, J (1967). "On shell solutions for masonry domes", International Journal of Solids and Structures, Vol. 3, pp. 227-241.

Heyman, J (1969). “The safety of masonry arches," International Journal of Mechanical Sciences, Vol. 11, pp. 363-385.

Kooharian, A (1952). "Limit analysis of voussoir (segmental) and concrete arches," Journal of the American Concrete Institute, Vol. 24, No. 4, pp. 317-328.

Lemos, J V (1998). "Numerical models for seismic analysis of monuments". Taller sobre desempeño sísmico de monumentos, Monument-98. Lisboa, Portugal, pp. K19-K36. 
Livesley, R K (1978). "Limit analysis of structures formed from rigid blocks," International Journal for Numerical Methods in Engineering, Vol. 12, pp. 1853-1871.

Livesley, R K (1992). “A computational model for the limit analysis of three-dimensional masonry structures," Meccanica, Vol. 27, pp. 161-172.

Lourenço, P B (1996). "Computational strategies for masonry structures", Tesis de doctorado, Universidad Técnica de Delft, Delft, Holanda.

Lourenço, P B (1998). "Sensitivity analysis of masonry structures", VIII Simposium Canadiense sobre Mampostería, Jasper, Canada, pp. 563-574.

Lourenço, P B y A Orduña (2000) "Análise e reforço da Igreja de São Francisco na Horta (Açores)" Informe CON-00-DEC/E-6, Departamento de Ingeniería Civil, Universidad de Minho, Guimarães, Portugal, 102 páginas.

Lourenço, P B (2002). "Computations on historic masonry structures". Progress in Structural Engineering and Materials, Vol 4, No. 3, pp. 301-319.

Nielsen, M P (1999). Limit analysis and concrete plasticity, segunda edición, CRC Press.

Orduña, A (2003), "Seismic assessment of ancient masonry structures by rigid blocks limit analysis," Tesis de doctorado, Universidad del Minho, Portugal, 154 pp. Disponible en www.civil.uminho.pt/masonry.

Orduña, A y P B Lourenço (2003). "Cap model for limit analysis and strengthening of masonry structures," Journal of Structural Engineering, ASCE, Vol. 129, No. 10, pp. 1367-1375.

Orduña, A y P B Lourenço (2005a). "Three-dimensional limit analysis of rigid blocks assemblages. Part I: Torsion failure on frictional interfaces and limit analysis formulation," International Journal of Solids and Structures, Vol. 42, No. 18-19, pp. 5140-5160.

Orduña, A y P B Lourenço (2005b). "Three-dimensional limit analysis of rigid blocks assemblages. Part II: Load-path following solution procedure and validation," International Journal of Solids and Structures, Vol. 42, No. 18-19, pp. 5161-5180.

Pegon, P, A Pinto y M Géradin (2001). "Numerical modeling of stone block monumental structures," Journal of Computer and Structures, Vol. 79, pp. 2165-2181.

Peña, F (2001). "Rigid element model for dynamic analysis of in-plane masonry structures," Tesis de doctorado, Politécnico de Milán, Italia.

Roedor, G M (2004). "Simulación numérica del comportamiento mecánico de la mampostería," Tesis de doctorado, Coordinación de Estudios de Posgrado, Universidad Nacional Autónoma de México, México DF, México.

Rots, J G (1988). "Computational modelling of concrete fracture", Tesis de doctorado, Universidad Técnica de Delft, Delft, Holanda.

SIMO, J C y J W Ju (1987). Strain- and stress-based continuum damage models. I: Formulation", International Journal of Solids and Structures, Vol 23, No 7, pp. 821-840. 